CONFERENCE SCENE

For reprint orders, please contact: reprints@futuremedicine.com

\title{
Current dementia research in Australia: a report from the Alzheimer's Australia 15th National Conference
}

Elizabeth Beattie*1, Elaine Fielding ${ }^{1}$, Maria O'Reilly ${ }^{1}$, Fred Graham ${ }^{1}$

\& Christine Neville ${ }^{2}$

\section{Alzheimer's Australia 15th National Conference, Hobart, Tasmania, Australia, 14-17 May 2013}

The Alzheimer's Australia 15th National Conference held on 14-17 May 2013 in Hobart (Tasmania, Australia) attracted a wide range of attendees, including people living with dementia, family caregivers, health professionals and researchers. The conference theme, The Tiles of Life Coloring the Future, invoking a vision of a better future for those affected by dementia, had seven subthemes: liberation, rehabilitation, leisure, service, creativity, wellbeing and research.

Natalie Jackson's (University of Waikato, New Zealand) eye-opening plenary (service subtheme) 'Population aging and Alzheimer's - an A-B-C approach' explored aging and dementia in developed societies. Over the next 20 years, population aging will lead to an extremely rapid increase, a doubling in fact, of the numbers of people with dementia, owing to the size and longer life expectancy of the baby-boom generation, exacerbated by the steeply curved increase in dementia prevalence with age. Over the same period, the fall in birth rates post-baby boom will lead to only a modest (a third) increase in the supply of working-age people, which is completely insufficient to cope with greatly increased service demands.

Jackson's proposed A-B-C response to this supply-demand crisis in developed countries was: accept that population aging will lead to significantly increased numbers of people with dementia and will affect all aspects of society; build a buffer by revisiting and revising policies (e.g., by considering the value of increased migration of young people); and collaborate and conserve, given that increasingly scarce resources will be spread among expanding numbers of people needing care, collaborative models of service delivery may be more effective compared with competitive ones.

Tony Broe (University of New South Wales [Sydney, Australia]/Neuroscience Research Australia [Sydney, Australia]) gave an important plenary (liberation subtheme), 'Brain health and dementia in urban Aboriginal Australians: outcomes from the Koori Growing Old Well Study', reporting on dementia prevalence among urban Aboriginal Australians [101]. Aboriginals have a young age distribution and a typically younger onset of dementia symptoms. However, as for the general population, the number of Aboriginals with
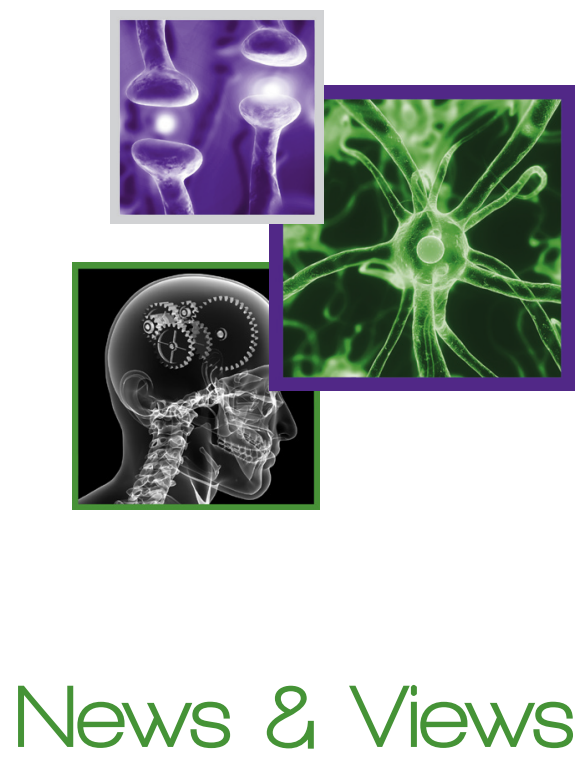

News

Journal Watch

Ask the Experts

Interview

Conference Scene

'Dementia Collaborative Research Centre, School of Nursing, Queensland University of Technology, Queensland,

Australia

${ }^{2}$ School of Nursing \& Midwifery, University of Queensland, Queensland, Australia

*Author for correspondence: elizabeth.beattie@qut.edu.au

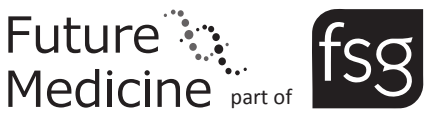


dementia is expected to increase rapidly over the next 20 years. Using a unique, community-based recruitment strategy, Broe's team screened over 300 urban Aboriginals aged 60 years or older, and found rates of dementia (21\% age adjusted) and mild cognitive impairment (MCI; 18\%) to be three-times higher than in the nonindigenous population, which is consistent with results from a recent study of remote area indigenous populations [1]. Among those with dementia, the percentage attributed to Alzheimer's disease (AD) was disproportionately high (56\%), as was that due to head trauma (12\%), compared with nonindigenous Australians. Among people with AD, in the Kgows Study, 40-50\% have sleep disturbance [101]. While approximately $5 \%$ of those with dementia exhibited signs of alcohol-related dementia, this figure was lower than expected. Factors associated with higher dementia risk included age, male gender, stroke, head injury, epilepsy, and a history of alcohol or drug use. Broe presented a comprehensive explanatory model of factors underpinning these results, including childhood risks (e.g., trauma, low education and epigenetics), which affect early adult risks (e.g., employment, education and head injury), which both affect later-life risks (e.g., alcohol, smoking, mental illness, obesity, heart disease and stroke). Describing this as a 'cascade of risk factors', he noted that interventions need to occur at all trajectory points to minimize the impact of dementia in indigenous Australians.

Michelle Bourgeois's (Department of Speech and Hearing Science, Ohio State University, $\mathrm{OH}$, USA) interesting (rehabilitation subtheme) plenary session 'Unlocking the silent prison: using visual cues to communicate with persons with dementia' focused on the use of visual cues to assist communication and function in people with dementia. Communication problems are common in dementia, leading to frustration, loss of ability to participate in daily tasks and social isolation. Bourgeois advocates the use of pictures and simple written sentences to enhance communication. A key message is that pictures and text together can be used in all stages of dementia. Although the text complexity requires simplification, she believes that reading is preserved late into the disease. In early disease, portable 'memory wallets' using pictures and cues can assist conversation, while in the later stages, 'memory books' can help to calm and engage. Bourgeois has trialed other uses for visual and written cues to help nursing home staff communicate effectively with residents with dementia. Cueing taps into preserved memory capacity by reducing the demands on declarative memory systems, enabling people to continue meaningful engagement for a much longer period.

Another interesting plenary session (wellbeing subtheme), 'Sleep well, think well: sleep-wake changes in neurodegenerative disease', was presented by Sharon Naismith (University of Sydney, Sydney, Austraila). As well as being important for mood, alertness, daytime functioning and cognition, Naismith contends that sleep could also be a modifiable risk factor for cognitive decline. She described the important relationship between sleep, memory formation and neural regeneration. Sleep-wake cycle changes occur as a natural part of the aging cycle after 40 years of age. Among people with AD, $40-50 \%$ have sleep disturbance, characterized by excessive daytime sleepiness, nocturnal insomnia and, for some, rapid eye movement sleep disorder - a particularly disturbing phenomenon whereby sufferers physically act out their dreams. Sleep disturbance also characterizes a high proportion (14-59\%) of people with MCI [2]. People with AD display a notable reduction in sleep spindles (the bursts of activity preceding the onset of stage 2 sleep, thought to be important for new memory formation) based on EEG readings.

Melatonin use in people with MCI has shown promise in improving sleep quality and cognitive function. In $\mathrm{AD}$, melatonin has more variable results; however, melatonin used in combination with light therapy arguably offers superior effects. Other nonpharmacological interventions with effectiveness for sleep regulation are morning or late afternoon (not near bedtime) exercise, good 'sleep hygiene' (e.g., a quiet environment, regular routines of retiring and rising, and maintaining suitable body temperature for sleep) and short daytime napping (30-45 min only). Light therapy has efficacy in advancing (morning exposure) or delaying (early-evening exposure) sleep onset. The take away message was that sleep-wake disturbances are a normal part of aging that are exacerbated in MCI and $\mathrm{AD}$, with their effect on cognition requiring further study.

Michael Woodward (University of Melbourne, Melbourne, Australia) gave an intriguing talk (research subtheme) titled 'Alzheimer's disease: the spectrum of clinical presentations about potential subtypes within AD'. His research into the clinical presentation and subsequent course 
of $\mathrm{AD}$, combined with neuroimaging, suggests the existence of multiple presentations described in four separate phenotypes. Types are distinguished by six characteristics: age of onset, genetics, early clinical features, clinical trajectory, neuroimaging/other biomarkers and pathology. The earliest clinical feature of amnestic AD is memory loss and early pathology can be detected in the limbic system, including the hippocampus. Logopenic aphasia or language AD is characterized by early language difficulties (e.g., with naming or word finding) [3]. This type shows damage in the temporal gyri and parietal lobules of the brain. Those falling into the visuospatial AD type can be younger (50-70 years of age) and display difficulties in facial and object recognition. Neuroimaging shows atrophy in the posterior cortical regions. The fourth type, behavioral- or frontal-variant $\mathrm{AD}$, is an older group with many typical $\mathrm{AD}$ clinical and neuroimaging features, combined with early behavioral or frontal features [4]. The behavioral characteristics of this type, such as aggression, could lead to a diagnosis of frontotemporal dementia. Distinguishing between the two may only be possible at the pathology stage, yet Woodward suggests that behavioral assessment may aid differentiation.

Brian Draper (University of New South Wales) gave a revealing overview of initial findings from the Hospital Dementia Services project [5] (service subtheme) in 'What influences outcomes of acute hospitalization of people with dementia?' This project examined outcomes for all people with dementia admitted to public hospitals in New South Wales over a year. Draper focused on associations between patient-related factors, hospital-related factors and patient outcomes. Significantly, for almost $50 \%$ of the admissions, dementia was not recorded as a diagnosis. Furthermore, approximately $94 \%$ of admissions were recorded as being for a reason other than dementia. Patients with dementia, compared with those without, experienced a higher prevalence of urinary tract infection, pressure areas, pneumonia, sepsis, delirium and failure to rescue. Certain patient-related factors (ill-defined conditions, surgical procedures, a principal diagnosis of dementia and younger-onset dementia) and hospital-related characteristics (visiting the geriatric service, visiting specialists, transferred within $48 \mathrm{~h}$ and an inner regional site) were associated with increased length of stay (LoS). Other patient-related characteristics (a high level of comorbidities, primary mental health and behavioral symptoms, age $<85$ years and readmission within 7 days) and hospital-related characteristics (90-100\% acute beds, a memory clinic visit, transferred from aged care and visiting mental health staff) were associated with decreased LoS. Two findings were unexpected: longer LoS among younger-onset dementia patients, and longer LoS in hospitals with visiting geriatricians and geriatric care specialists. These findings may indicate that LoS data might not necessarily reflect quality of care. To more accurately predict quality of care and outcomes, principle component analysis was used, which yielded four main hospital characteristics as important predictors: hospital cultures; staff leadership; the presence of skilled (specialist) staff; and the hospital environment. Hospitals rating higher in these areas produced better outcomes for patients with dementia.

Elizabeth Beattie (Queensland University of Technology, Queensland, Australia) reported on the initial findings from a longitudinal study of perceived stigma in a presentation titled 'Dementia, stigma, health and wellbeing - are there connections?' (wellbeing subtheme) conducted by a team from the Dementia Collaborative Research Centre: Carers and Consumers. Mental illnessrelated stigma has been associated with higher depressive symptoms and a range of other poor health and wellbeing outcomes. However, little research has explored stigma in people with dementia and their primary caregivers. This study ( $\mathrm{n}=19$ dyads, persons with dementia and caregivers) collected data at baseline, 6 and 12 months, with a pending final data point at 18 months [6]. Perceived stigma (PS) was measured using the Stigma Impact Scale. A battery of health and wellbeing measures and individual and dyad interviews were also undertaken. Importantly, at the 6-month point, higher PS was associated with higher anxiety levels and self-reported depressive symptoms and with lower self-esteem, self-reported physical health, sense of personal control and perceived levels of social support. Interestingly, PS for the person with dementia was not linked to the level of cognitive impairment, type of dementia symptoms or caregiver dementia knowledge. Caregivers' PS level was not associated with most aspects of health and wellbeing in the persons with dementia, such as anxiety and physical health. However, there was a significant association between PS of family caregivers related to higher reported recreational and social activities of the person with dementia and also when those with memory loss had 
lower levels of personal control. In other words, aspects related to memory loss and behavior that highlighted impairment and differences in public situations seem to be perceived as stigmatizing by family caregivers. These initial findings suggest there may be important perspectives to be considered very early in the trajectory of the disease to help facilitate increased community involvement, interaction and socialization to reduce stigma and facilitate dementia-enabling communities.

A concurrent session (wellbeing subtheme) titled 'The prevalence of behavioural and psychological symptoms of dementia in people living at home with their families', was presented by Christine Neville (University of Queensland, Australia), representing a second research team from the Dementia Collaborative Research Centre: Carers and Consumers. This study is the first to determine the prevalence of behavioral and psychological symptoms of dementia (BPSDs) in community-dwelling people with dementia in Australia. BPSDs can be highly distressing to both people with dementia and caregivers, and are key factors in early nursing home placement.
Data from the cross-sectional phone and internet survey ( $\mathrm{n}=326$ family carers) found that $94 \%$ of people with dementia exhibited at least one BPSD and more than half (53\%) exhibited five or more BPSDs during the week prior to survey completion. People with more severe dementia had higher rates of BPSD and younger carers reported higher frequencies of BPSDs. These rates are very high and Neville stressed the need for family and health workers to be alert for BPSDs in view of the substantial negative implications both for the individual with dementia and their carers.

\section{Financial \& competing interests disclosure}

The authors have no relevant affiliations or financial involvement with any organization or entity with a financial interest in or financial conflict with the subject matter or materials discussed in the manuscript. This includes employment, consultancies, honoraria, stock ownership or options, expert testimony, grants or patents received or pending, or royalties.

No writing assistance was utilized in the production of this manuscript.

\section{References}

1 Smith K, Flicker L, Lautenschlager T et al. High prevalence of dementia and cognitive impairment in Indigenous Australians. Neurology 71, 1470-1473 (2008).

2 Naismith S, Rogers N, MacKenzie J, Norrie L, Lewis S. Sleep well, think well: sleep-wake disturbance in mild cognitive impairment. J. Geriatr. Psychiatry Neurol. 23(2), 123-130 (2010).
3 Gorno-Tempini ML, Brambati SM et al. The logopenic/phonological variant of primary progressive aphasia. Neurology 71(16), 1227-1234 (2008).

4 Woodward M, Jacova C, Black S, Kertesz A, MacKenzie I, Feldman H; the ACCORD Investigators. Differentiating the frontal variant of Alzheimer's disease. Int. J. Geriatr. Psychiatry 25, 732-738 (2010).

5 Australian Institute of Health and Welfare. The Hospital Dementia Services Project: a Study
Description. Australian Institute of Health and Welfare, Canberra, Australia (2011).

6 Bergener S, Berger B. Measuring perceived stigma in persons with progressive neurological disease. Dementia 7(1), 31-53 (2008).

\section{- Website}

101 Neuroscience Research Australia. Koori Growing Old Well Study. www.neura.edu.au/aboriginal-ageing 